\title{
Mature Bone-Producing Osteoblasts Alter Gene Expression of Metastasis Related Genes in Prostate Cancer Cells
}

\author{
Haakon Skogseth ${ }^{1 *}$, Marius Dybwad ${ }^{1}$, Arnar Flatberg ${ }^{1}$ and Jostein Halgunset ${ }^{1,2}$ \\ ${ }^{1}$ Department of Laboratory Medicine, Children's and Women's Health, Faculty of Medicine, Norwegian University of Science and Technology, Trondheim, Norway \\ ${ }^{2}$ Department of Pathology and Molecular Genetics, St. Olav's Hospital, Trondheim, Norway
}

\begin{abstract}
Background: Prostate cancers often metastasize to bone. It is hypothesized that interactions between osteoblasts and cancer cells lead to establishment of prostate cancer skeletal metastasis.

Methods: As a result of co-culture between undifferentiated and differentiated osteoblasts and PC-3 and DU-145 prostate cancer cells, gene expressional changes were determined with microarray technology, both Affymetrix ${ }^{\circ}$ and Illumina $®$ protocols. Characterization of osteoblast differentiation was performed using alizarin red staining of calcium, saffron staining of collagen fibers and quantitative real-time PCR of differentiation markers (RUNX2, Osteocalcin and Alkaline phosphatase).

Results: The Affymetrix ${ }^{\circ}$ protocol demonstrated that co-culturing of PC-3 cells with differentiated osteoblasts resulted in significantly more expressional changes in the cancer cells than co-culturing with the undifferentiated osteoblasts. The data generated from the Illumina Beadchip platform on DU-144 cells demonstrate a significant larger differential expression (399 reporters) in co-culture with undifferentiated osteoblast cells compared to the differentiated (35 reporters). Morover, this is in contrast to PC-3 cells where co-culture with differentiated osteoblasts dominates the differential expression with 340 reporters while there are only 11 in the case of undifferentiated. However, the Go-Term "Regulation of caspase" (GO:0043281) was found in common in both Affymetrix $®$ and Illumina ${ }^{\circledR}$ array protocols.

Conclusion: Interactions between prostate cancer cells and mature bone-producing osteoblasts resulted in gene expressional changes that may increase cells' ability to establish bone metastasis. The potential for inducing expressional changes seems to be crucial dependent of the differentiation status of osteoblasts.
\end{abstract}

Keywords: Gene expression; Co-culture; Prostate cancer; PC-3; DU145 osteoblast; hFOB1.19

Abbreviations: NMC: Norwegian Microarray Consortium; PLS: Partial Least Squares

\section{Introduction}

Most prostate cancers grow slowly as an indolent disease. However, some show an aggressive behavior with rapid growth and widespread metastases, mainly to the skeleton, lymphatic nodes, lung and liver [1]. Among patients with metastatic prostate cancer, bone lesions will be found in $90 \%$, a fact which underscores the particular propensity of this disease to skeletal spread [2,3]. Unfortunately, patients with bone metastases have an average survival of only 9 to 12 months $[4,5]$. Thus, a better understanding of the underlying mechanisms might be of great significance to future patients.

It has been hypothesized that the bone microenvironment exerts an attractive effect on prostate cancer cells [6,7], involving a complex interplay between the prostate epithelial tumor cells and various bone tissue components. Studies have shown that homogenate from bone tissue increased prostate cancer cells' ability to invade in vitro, while homogenate of brain tissue showed no such effect. However, the details of this interaction are far from elucidated. In normal bone a mixture of cellular elements is found, comprising osteoblasts, osteoclasts, fibroblast-like cells and endothelium, as well as an array of blood cells and their precursors. The extracellular matrix composed of collagen type I, osteopontin, osteocalcin, osteonectin and hydroxyapatite. Several of these various components have been suggested to affect invasion, proliferation and survival of prostate cells in bone tissue. Thus, it has been shown that an increased malignant potential can be induced by osteonectin [8]. It has been reported that growth factors such as epidermal growth factor (EGF), transforming growth factor beta (TGF- $\beta$ ) and insulin growth factor (IGF) I/II have such an effect $[9,10,11]$. Moreover, chemotactic cytokines, so-called chemokines, have been shown to induce directed movement in cancer cells [12,13]. For instance, stroma derived factor 1 (SDF-1), also denoted CXCL12, and its corresponding membrane receptor CXCR4, confer a more invasive phenotype on selected prostate cancer cell lines. It is highly suggestive that SDF-1 is expressed both by osteoblasts and bone endothelial cells, as well as by bone stromal fibroblasts, while its receptor is present in several cell lines derived from prostate cancer [14]. It has been proposed that prostate cancer cells' extravasation and movement into bone marrow space, is enhanced by a selective adhesion between the cancer cells and bone marrow endothelial lining [5]. Studies have shown that the integrin subunits $\beta 1$ and $\beta 3$ are participators in such type of adhesion. In view of the rich expression of these molecules by prostate cells, it is reasonable to suppose a role of integrins in the skeletal homing of prostate cancer cells. Interestingly, the binding of prostate cancer cells to bone marrow endothelial cells is much more effective than their adhesion to endothelium from the umbilical cord [15].

*Corresponding author: Haakon Skogseth, Department of Laboratory Medicine Children's and Woman's Health Faculty of Medicine, Norwegian University of Science and Technology, St. Olav's Hospital, 7006 Trondheim, Norway, Tel: +47 72573081; Email: haakon.skogseth@ntnu.no

Received November 17, 2010; Accepted December 04, 2010; Published December 06, 2010

Citation: Skogseth H, Dybwad M, Flatberg A, Halgunset J (2010) Mature BoneProducing Osteoblasts Alter Gene Expression of Metastasis Related Genes in Prostate Cancer Cells. J Carcinogene Mutagene 1:111. doi:10.4172/21572518.1000111

Copyright: (c) 2010 Skogseth $\mathrm{H}$, et al. This is an open-access article distributed under the terms of the Creative Commons Attribution License, which permits unrestricted use, distribution, and reproduction in any medium, provided the original author and source are credited. 
The establishment of secondary prostate cancer deposits in bone may be promoted by specific changes of the tumor cells' behavior, rendering them more similar to those of skeletal tissue and thus becoming better equipped for survival in bone tissue [16]. Thus, the interaction between prostate cancer cells and bone tissue components may induce a conversion of the former, making them assume a gene expression profile more similar to that of bonespecific cells [17]. A number of studies have dealt with the mutual signaling between prostate cells and osteoblasts, mainly focusing on alterations in the latter. Far less is known about the possible influence of osteoblasts on the prostate cancer cells. The picture is further complicated by the fact that cancer cells arriving in the bone marrow, may encounter both mature bone-producing osteoblasts (differentiated) and immature osteoprogenitor-like osteoblasts (non-differentiated). It is therefore of interest to know whether the differentiation status of the osteoblasts may modulate the way they interact with prostate cancer cells.

\section{Materials and Methods}

\section{Cell lines and cultures}

The human prostatic carcinoma cell lines PC-3 and DU-145 and the human fetal osteoblastic cell line hFOB1.19 (CRL-11372) were obtained from the American Type Culture Collection (ATCC). PC-3 [18] was originally derived from a hormone-independent prostatic skeletal metastasis, does not express the prostate specific antigen (PSA), and tends to yield osteolytic skeletal metastasis when grafted into mice. DU-145, which was originally grown out of brain tissue from a prostate cancer metastasis, does not express PSA. HFOB1.19 Harris et al. [19] was originally derived from a spontaneous miscarriage, and was rendered immortal by transfection with a temperature sensitive variant of the large T-antigen from the Simian Virus (SV40) and the antibiotic resistance-coding neo-gene. The cell line proliferates rapidly at $33.5^{\circ} \mathrm{C}$ (permissive temperature), but the proliferation is reduced if the temperature is increased, and is nearly abolished at $39^{\circ} \mathrm{C}$ (restrictive temperature). HFOB1.19 cells show an osteoprogenitor-like phenotype, but have the ability to differentiate into mature osteoblasts. Osteoblastic differentiation is induced by many factors, like incubation temperature, time in culture, degree of confluence and inductive media supplements. All cell lines were grown in $75 \mathrm{~cm} 2$ tissue culture flasks (Sarstedt, Germany) in a humidified environment containing 5\% CO2 in air. PC-3 and DU-145 were maintained at $37^{\circ} \mathrm{C}$ in $1: 1 \mathrm{Ham}$ 's $\mathrm{F} 12$ medium and Dulbeccos modified Eagle's medium without phenol-red, supplemented with $10 \%$ fetal bovine serum and $2 \%$ penicillin/streptomycin. HFOB 1.19 was maintained at $33.5^{\circ} \mathrm{C}$ in the same medium as PC-3 and DU-145, but with Geneticin G418 $(300 \mu \mathrm{g} / \mathrm{ml})$ instead of penicillin/streptomycin. All the media reagents were obtained from Sigma (Sigma-Aldrich, USA). All cell lines were routinely tested for the presence of mycoplasma with real-time PCR using the broad-range LightCycler Mycoplasma Primer Kit (Search LC GmbH, Germany), and were found to be free of infection.

\section{Differentiation of hFOB1.19 cells}

HFOB1.19 cells (200 000 cells/well) were seeded in 6-well tissue culture plates and cultured at $33.5^{\circ} \mathrm{C}$ until $100 \%$ confluence (day 0 ), after which the temperature was raised to $37^{\circ} \mathrm{C}$. In half of the wells, the medium was supplemented with the following differentiation inducing mixture: dexamethasone $(10-8 \mathrm{M})$, ascorbic acid $(100 \mu \mathrm{g} / \mathrm{ml})$, $\beta$-glycerophosphate $(10 \mathrm{mM})$ and 1,25-dihydroxy-vitamin D3 (10-8M) $[20,21,22]$. All supplements were obtained from Sigma. The culture medium was changed every 72 hours and plates were collected for calcium-staining, collagen-staining and real-time PCR at four different times during the cultivation (day 0 , day 6 , day 12 and day 18 ).

\section{Visualization of calcium deposition}

Calcium in mineralized matrix was stained with alizarin red, using a previously described protocol with only minor modifications [23]. Briefly, the medium was aspired, and the cells were washed with phosphate buffered saline (PBS) (2X, room temperature), fixed with $3 \%$ paraformaldehyd (PFA) (10 minutes, on ice), rinsed carefully with PBS (1X, room temperature) and de-ionized $\mathrm{H} 2 \mathrm{O}$ (1X, room temperature), stained with alizarin red $(1 \mathrm{X}, 40 \mathrm{mM}, \mathrm{pH} 4.2,30$ minutes, room temperature), washed with de-ionized $\mathrm{H} 2 \mathrm{O}$ (5X, room temperature) and finally incubated with PBS (1X, 15minutes, room temperature) to remove unspecific staining. All the reagents were obtained from Sigma. The results were documented using a digital SLR camera or with an Axiovert 200M (Zeiss, Germany) inverted microscope equipped with an AxioCam MRm (Zeiss, Germany) digital camera.

\section{Visualization of collagen in osteoid}

Collagen in osteoblast cultures was stained using saffron. The culture dishes were washed with PBS (1X, room temperature), dehydrated in $80 \% \mathrm{EtOH}$ (1X, room temperature), dehydrated in absolute EtOH (1X, room temperature), stained with saffron $(5 \mathrm{mg} /$ $\mathrm{ml})$ in absolute $\mathrm{EtOH}(1 \mathrm{X}$, room temperature) and finally de-stained using absolute EtOH (3X, room temperature). Absolute EtOH was obtained from Arcus (Arcus, Norway) and saffron was obtained readymade directly from the local histology laboratory. The results were documented using a digital SLR camera.

\section{Quantization of osteoblast differentiation markers}

Real-time PCR was used to determine the amount of transcripts for three markers of osteoblastic differentiation, RUNX2, osteocalcin (OC) and alkaline phosphatase (ALP). Total-RNA was isolated from the cells using a MagNA Pure LC nucleic acid isolation robot (Roche Diagnostics GmbH, Germany) combined with the MagNA Pure LC RNA isolation Kit-High Performance (Roche Diagnostics $\mathrm{GmbH}$, Germany). The amount of RNA was measured using a ND-1000 spetrophotometer (NanoDrop Technologies, USA), and the RNA integrity was assessed with capillary gel electrophoresis in a 2100 Bioanalyzer and RNA 6000 Nano Kit (both from Agilent Technologies, USA). Reverse transcription of mRNA to cDNA was done with a polydT primer using total-RNA $(1 \mu \mathrm{g})$ and Transcriptor First Strand cDNA synthesis Kit (Roche Diagnostics GmbH, Germany). Real-time PCR was conducted in glass capillaries $(20 \mu \mathrm{l})$ in a LightCycler 2.0 (Roche Diagnostics GmbH, Germany) instrument using the LightCycler FastStart DNA MasterPLUS HybProbe kit (Roche Diagnostics $\mathrm{GmbH}$, Germany), as a calibrator-normalized relative quantization using the synthesized cDNA $(5 \mu \mathrm{l}, 10 \mathrm{ng} / \mu \mathrm{l})$. All real-time PCR reactions in this experiment were done in triplicates. $\beta$-Actin was tested and found suitable as a housekeeping gene since the expression did not

\begin{tabular}{|l|l|}
\hline Gene & Forward/Reverse primer \\
\hline RUNX2 & $\begin{array}{l}\text { CCTAggCgCATTTCAgATgAT/ } \\
\text { TAgCgTgCTgCCATTCgA }\end{array}$ \\
\hline ALP & $\begin{array}{l}\text { ACggACTCCTgACCCTTgA/ } \\
\text { TCCgCAggATCTggATgg }\end{array}$ \\
\hline OC & $\begin{array}{l}\text { AAggTgCAgCCTTTTgT/ } \\
\text { AAgggTgCCTggAgAggA }\end{array}$ \\
\hline$\beta$-Actin & $\begin{array}{l}\text { AgCCTCgCCTTTCCgA/ } \\
\text { CTggTgCCTggggCg }\end{array}$ \\
\hline
\end{tabular}

Table 1: Sequences of the primers for the differentiation markers and the housekeeping gene used in real-time PCR. 
appreciably change with the different culture lengths or conditions used. Primers and probes for the differentiation markers (RUNX2, OC and ALP) and the housekeeping gene ( $\beta$-Actin) were obtained from TIB Molbiol (TIB Molbiol, Germany). The sequences of the primers and probes are presented in Table 1. A reference material (calibrator) was used to make a standard curve for each of the differentiation markers and the housekeeping gene and included in each run, thus enabling compensation for different PCR-efficiencies between runs. The calibrator was cDNA synthesized from total-RNA isolated from hFOB1.19 cells grown to $100 \%$ confluence (day 0 ) under standard culture conditions. The PCR products were checked with melting point analysis in the LightCycler 2.0, capillary gel-electrophoresis in the Bioanalyzer 2100 using a DNA 1000 kit (Agilent Technologies, USA) and sequencing with the CEQ 8800 Genetic Analyzer (Beckman Coulter, USA) using a GenomeLab Dye Terminator Cycle Sequencing Kit (Beckman Coulter, USA).

\section{Co-culture model}

PC-3 And DU-145 cells (200 000 cells/insert) were seeded into tissue culture inserts containing a porous $(0.4 \mu \mathrm{m})$ polyethylene terephtalate (PET) membrane (BD Falcon, USA) and grown to $100 \%$ confluence in normal growth medium at $37^{\circ} \mathrm{C}$ before transfer to the co-culture system. HFOB1.19 cells (200 000 cells/well) were seeded into 6-well tissue culture plates and grown to $100 \%$ confluence (day 0 ) in normal growth medium at $33.5^{\circ} \mathrm{C}$, thus yielding hFOB1.19 cells with a osteprogenitor-like phenotype. Half of the wells were used in the co-culture at day 0 , while the rest were further differentiated into mature bone-producing osteoblasts by changing the temperature to $37^{\circ} \mathrm{C}$, with media supplements as described above, and grown for additional 18 days (day 18). Inserts containing PC-3 cells were then placed in wells containing osteoprogenitor-like hFOB1.19 cells (undifferentiated), mature bone-producing hFOB1.19 cells (differentiated), or no hFOB1.19 cells (control), and further cultured for 72 hours at $37^{\circ} \mathrm{C}$ in normal growth medium without serum or antibiotics. The co-culture model thus allowed free diffusion of soluble substances between the chambers, without any physical contact between the two cell types. All inserts and wells were carefully washed with PBS before placing them together. The medium was changed every 72 hours for growth and differentiation, and every 36 hours during co-culture.

After the co-culture period total-RNA was isolated from the PC-3 and DU-145 cells and its amount and quality controlled using the same procedure as the one described above for osteoblasts. Total-RNA isolated from two parallel PC-3 and DU-145 containing inserts was pooled together $(2 \mu \mathrm{g}+2 \mu \mathrm{g})$, thus producing a total of 24 samples, comprising three samples from PC-3 and DU-145 cells co-cultured with un-differentiated hFOB1.19 cells linked to three corresponding controls, and three samples from PC-3 and DU-145 cells co-cultured with differentiated hFOB1.19 cells linked to three corresponding controls.

\section{Gene expression profiling}

Affymetrix protocol: cDNA was synthesized from 100ng totalRNA using the two-cycle cDNA Synthesis Kit, Sample Cleanup Module Kit, Eucaryotic Poly-A RNA Kit and IVT Labeling Kit, according to the producer's instructions (Affymetrix, USA). C-RNA synthesis was performed by MEGAscript T7 Kit obtained from Ambion (Ambion, USA). The gene expression profile was then obtained in each of the 12 samples by loading cDNA onto GeneChip HGU133 Plus 2.0 whole-genome microarrays (Affymetrix, USA). The microarray service was provided by the Norwegian Microarray Consortium (NMC) at the national technology platform, and supported by the functional genomics program (FUGE) in the Research Council of Norway.

Illumina protocol: We used the Illumina TotalPrep RNA amplification Kit (Ambion Inc., Austin, TX) to amplify RNA for hybridization on Illumina BeadChips. To synthesize first strand cDNA by reverse transcription, we used totalRNA from each sample collected above. Following the second strand cDNA synthesis and cDNA purification steps, the in vitro transcription to synthesize cRNA was prepared overnight for 12 hours. The gene expression profiles were measured on DU-145 and PC-3 cells using Illumina Human HT12 v3 Expression BeadChip (Illumina, San Diego, CA), which enables genome-wide expression analysis (more than 480000 transcripts) of 12 samples in parallel on a single microarray.

RNA quality measurement: RNA concentration and quality were determined using a NanoDrop ND-1000 spectrophotometer (NanoDrop Technologies, Wilmington, DE) and Agilent 2100 Bioanalyzer (Agilent Technologies, Palo Alto, CA), which calculates an RNA integrity number (RIN). The RIN values of RNA samples used for cRNA amplification ranged from rin-min to rin-max, indicating good quality of RNA samples.

\section{Data analysis}

Affymetrix data analysis: Data analysis was performed using Robust Multichip Analysis (RMA) [24] Partial Least Squares (PLS)statistics [25] and the Limma software package for R [26]. Gene annotation and gene ontology were obtained using NMC's Annotation Tools and Explore Gene Ontology (eGOn) software available from GeneTools [27]. The data analysis service was provided by the NMC at the national technology platform, and supported by the FUGE program in the Research Council of Norway. The raw data and the normalized data from all the microarrays in this experiment were uploaded into the ArrayExpress (EBI, UK) database using the MIAME standard, thus providing online access to the complete dataset and a detailed description of the assay properties.

The reporter lists generated from prostate cancer cells co-cultured with the differentiated osteoblasts were loaded into GeneTools and annotated with GO-terms. A mutually exclusive target-target test (Fisher's exact test, $\mathrm{p}<0.01$ cut-off) comparing the up-regulated and down-regulated reporter lists was done in eGOn. The results show whether gene classes associated with specific biological processes had predominantly up-regulated or down-regulated reporters.

Illumina data analysis: Raw data were exported from the Illumina Genomestudio software to R using the Bioconductor package 'lumi` . The data were quantile normalized and $\log 2$ transformed. The groups were compared using a t-test with empirical Bayes correction from the Bioconductor package 'limma' [25]. The fold change was used to demonstrate changes in average gene expressions between studied groups. Statistical analyses were performed using the false discovery rate (FDR) with a significance threshold of 0.01 . The reporter lists generated from prostate cancer cells co-cultured with the differentiated osteoblasts were loaded into GeneTools and annotated with GO-terms. A mutually exclusive target-target test (Fisher's exact test, $\mathrm{p}<0.01$ cut-off) comparing the up-regulated and down-regulated reporter lists was done in eGOn. The results show whether gene classes associated with specific biological processes had predominantly up-regulated or down-regulated reporters. 

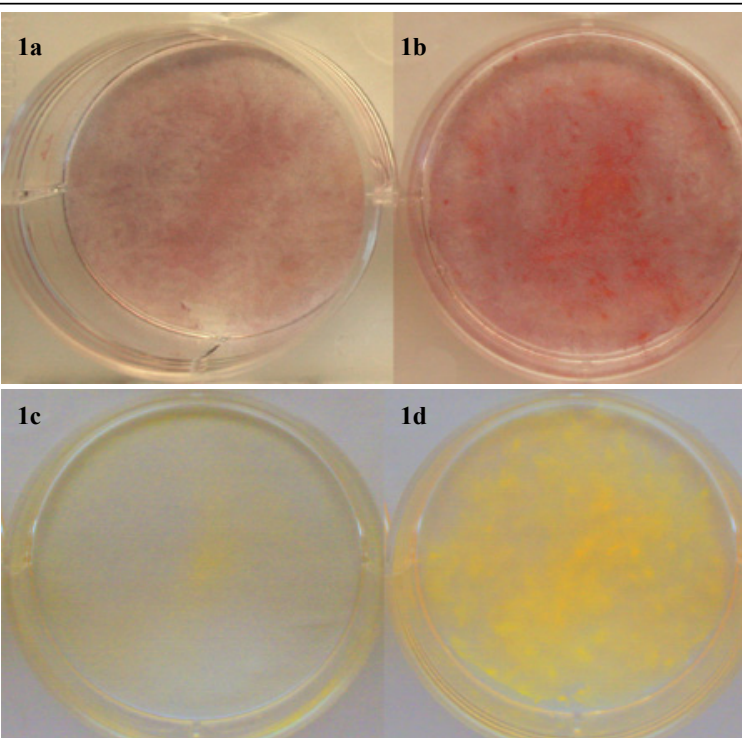

Figure 1: The HFOB1.19 cell line showed considerable more calcium staining (1b) when grown in differentiation media for 18 days compared to control (1a). Osteoblasts also expressed much more collagen after the incubation period (1d) compared to control (1c)

\section{Results}

Osteoblasts may be induced to secrete bone-specific extracellular matrix in culture

Calcium-staining with alizarin red and collagen-staining with saffron became detectable in confluent HFOB1.19 cultures around day 6 after change to differentiation-inducing conditions. Thereafter, both parameters gradually increased with further culture until 18 days, indicating the transition to a mature phenotype actively producing bone-specific extracellular matrix. Staining was also detected in wells cultured without the inductive supplements, but the presence of differentiation stimulatory factors led to more intense staining with earlier onset. Representative images of wells from staining analyses are presented in Figure 1.

\section{Osteoblasts may be induced to increase their expression of differentiation markers in culture}

The results from the calibrator-normalized relative quantization of RUNX2, ALP and OC with real-time RT-PCR showed that the expression of differentiation markers gradually increased with time. Although the different markers showed slightly different kinetics of expression increase, a significantly increased expression was demonstrated for all three markers at day 18 . However, the expression could be further stimulated by the addition of differentiation inductive supplements, which made the process accelerate. The gene expression data are depicted in Figure 2. Quality controls of the total-RNA samples before real-time RT-PCR showed 260/280- and 260/230-ratios of 2.0 $(+/-0.2$ ), RNA integrity numbers (RIN) $\geq 9.7$ and $18 \mathrm{~S} / 28 \mathrm{~S}$-ratios $\geq 2.0$ for all samples analyzed, indicating that the total-RNA samples had good purity and contained un-degraded RNA. Specific amplification during the RT-PCR reactions was confirmed by melting curve analysis and further by purifying the PCR products (PCR clean-up kit, Roche Diagnostics) prior to capillary gel electrophoresis (Agilent Bioanalyzer 2100, Agilent, USA) using the DNA 1000 kit (Agilent, USA).
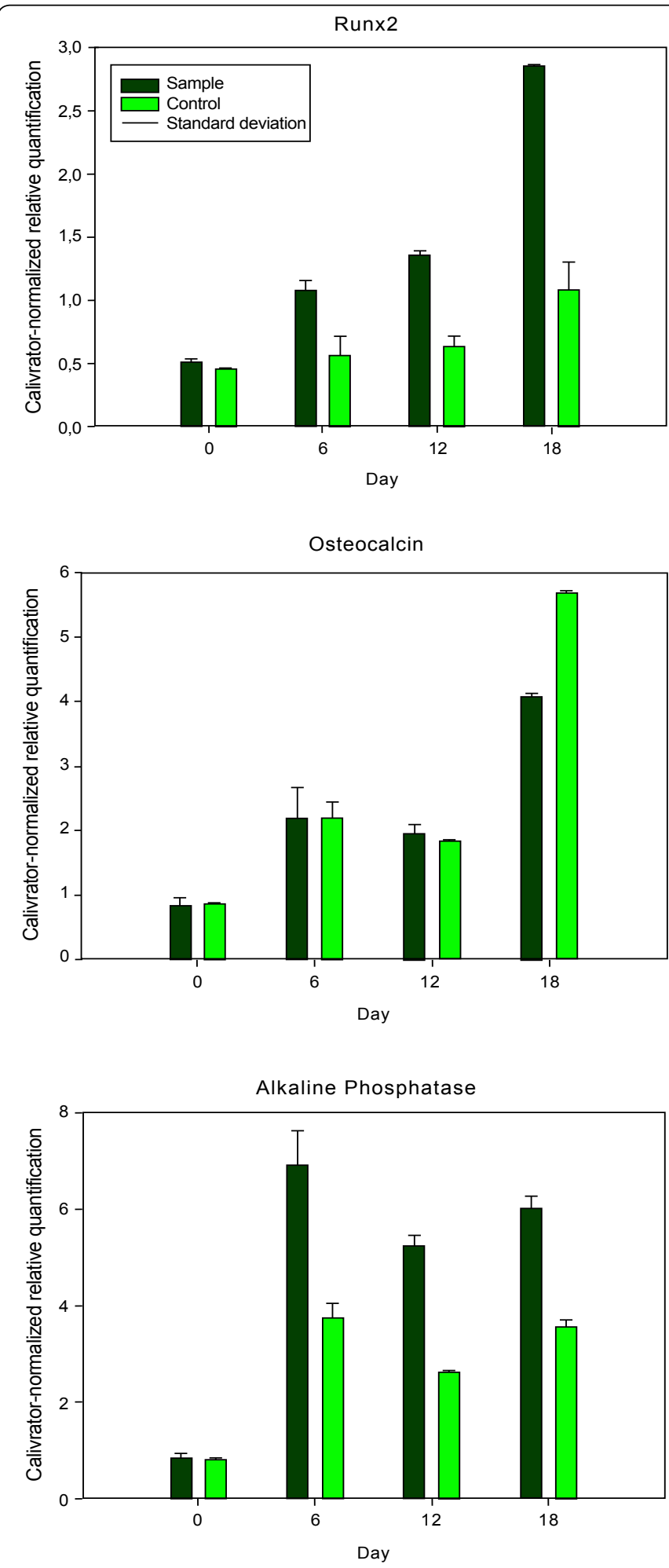

Figure 2: Results from the calibrator-normalized relative quantization analysis of the differentiation markers RUNX2, ALP and OC with real-time qRT-PCR. Summarized osteoblasts grown with differentiation factors showed a higher level of all three differentiation markers. RUNX2 and OC showed a time dependent gene expression, while the ALP gene was high expressed already at day 6 . In agreement with earlier reports, an increased time dependent differentiation was also induced in control cells (grown without differentiation factors). This differentiation could be explained by the osteoblasts' ability to spontaneous differentiate as result of high confluence in growth chambers. 


\begin{tabular}{|c|c|}
\hline GO-term & Function \\
\hline 0006917 & Induction of apoptosis \\
\hline 0043068 & Positive regulation of programmed cell death \\
\hline 0008633 & Activation of pro-apoptotic gene products \\
\hline 0043281 & Regulation of caspase activity \\
\hline 0008632 & Apoptotic program \\
\hline 0007160 & Cell-matrix adhesion \\
\hline
\end{tabular}

Table 2: Affymetrix protocol. The data generated from the Affymetrix® microarray analysis revealed that the differentiated, bone-matrix-producing hFOB1.19 osteoblasts affected the gene expression in PC-3 cells substantially more than the osteprogenitor-like, undifferentiated osteoblasts did. Interestingly, filtered GO-data showed specific gene classes which was affected by the co-culture between PC-3 cells and differentiated osteoblasts. The genes belonging to the gene class are predominantly repressed by co-culturing, mainly positive regulate apoptosis and cell adhesion.

\begin{tabular}{|l|l|l|l|l|}
\hline Type of regulation & $\begin{array}{l}\text { DU-145 } \\
\text { Differentiated versus control }\end{array}$ & $\begin{array}{l}\text { DU-145 } \\
\text { Undifferentiated versus control }\end{array}$ & $\begin{array}{l}\text { PC-3 } \\
\text { Differentiated versus control }\end{array}$ & $\begin{array}{l}\text { PC-3 } \\
\text { Undifferentiated versus control }\end{array}$ \\
\hline Repressed & 14 & 141 & 190 & 1 \\
\hline No change & 11568 & 11208 & 11267 \\
\hline Increased & 25 & 258 & 150 & 11596 \\
\hline
\end{tabular}

Table 3: Illumina protocol: The data generated from the Illumina Beadchip platform on DU-144 cells demonstrate a significant larger differential expression (399 reporters) in co-culture with undifferentiated osteoblast cells compared to the differentiated ( 35 reporters). This is in contrast to PC-3 cells where co-culture with differentiated osteoblasts dominates the differential expression with 340 reporters while there are only 11 in the case of undifferentiated.

\begin{tabular}{|l|l|l|l|}
\hline Gene symbol & Repressed & Increased & Function \\
\hline BIRC5 & $\mathrm{x}$ & & Inhibitor of apoptosis \\
\hline BAX & $\mathrm{x}$ & & Pro-apoptotic mediator \\
\hline VCP & $\mathrm{x}$ & & Vesicle transport and fusion \\
\hline TNFRSF10B & & $\mathrm{x}$ & Intracellular death domain \\
\hline TNFRSF10A & & $\mathrm{x}$ & Intracellular death domain \\
\hline CYCS & & X & Activates the apoptotic initiator procaspase 9 \\
\hline
\end{tabular}

Table 4: Genes in the Go-Term "Regulation of caspase" (GO:0043281) which were found in common in both Affymetrix ${ }^{\circledR}$ and Illumina ${ }^{\circledR}$ array protocols analyzed in PC-3 cells. No such changes were found in DU-145 cells.

\section{Osteoblasts' effect on cancer cell gene expression depends on their differentiation status}

The data generated from the Affymetrix ${ }^{\circledR}$ microarray analysis revealed that the differentiated, bone-matrix-producing hFOB1.19 osteoblasts affected the gene expression in PC-3 cells substantially more than the osteoprogenitor-like, undifferentiated osteoblasts did. By conventional statistics for the calculation of p-values adjusted for false discovery rate (FDR), a reporter list was generated (using $\mathrm{p}<0.01$ FDR cut-off) containing 4637 up-regulated reporters and 4818 down-regulated reporters in prostate cancer cells co-cultured with differentiated osteoblasts, as contrasted with only 5 up-regulated reporters and 2 down-regulated reporters in prostate cancer cells cocultured with undifferentiated osteoblasts.

The data generated from the Illumina Beadchip platform on DU144 cells demonstrate a significantly larger differential expression (399 reporters) in co-culture with undifferentiated osteoblast cells compared to the differentiated (35 reporters). This is in contrast to PC-3 cells where co-culture with differentiated osteoblasts dominates the differential expression with 340 reportes while there are only 11 in the case of undifferentiated (Table 3). However, no expressional changes associated with apoptosis in DU-145 could be found.

\section{Expression of cancer relevant genes is altered in prostate cancer cells by co-culture with differentiated osteoblasts}

With the Affymetrix ${ }^{\circledR}$ protocol, the GeneTools method (GO) showed that a number of gene classes were noticeably affected by o-culture model (Table 2). It is interesting that gene classes associated with the regulation of apoptosis in PC-3 cells seem to be affected by co-culture with differentiated osteoblasts. In addition, the cellmatrix adhesion gene class was noticeable affected, with repression of several adhesion molecules.

With the Illumina ${ }^{\circledR}$ array protocol, the GO-analysis reveals specific Gene Ontology terms over-represented in the list of significant reporters. There was no significant overlap between the results of the Illumina and Affymetrix platform when considering down- and up-regulated lists of reporters separately, however, when considering the regulation jointly as a list of differential regulated reporters the Go-Term `Regulation of caspase` (GO:0043281) was found in common. Of 41 genes belonging to this GO, 6 genes were affected by co-culturing (Table 4).

\section{Discussion}

Recent advance in prostate cancer research has led to an increasing body of knowledge, but key pieces are still lacking in the puzzle. Advances in clinical treatment of patients have up to now been achieved by optimization of available conventional therapies which have been used for many years. Prostate cancer is deceptively refractory to conventional cancer drugs, which have mainly been conceived as inhibitors of cell division. Considering the large number of deaths caused by this disease, a search for alternative therapeutic targets is highly warranted. A better understanding of the complex mechanisms governing the properties of cancer cells and their interactions with the rest of the organism is expected to be crucial in this respect. In order to metastasize to the skeleton, prostate cancer cells must detach from the primary tumor site, move to the location, and attach to bone tissue, whereupon they must survive and continue to proliferate and extend into the available space in the new environment. All these steps probably involve several events, some of which may constitute potential targets for efficient pharmaceutical manipulation.

Apparently several cell types are involved in prostate cancer cells' homing, but there is evidence that osteoblasts act as key triggers for the spread to bone, due to the fact that prostate cancer often forms osteoblastic metastases. Osteoblasts build bone, and in this process they may secrete substances that could potentially affect cancer cells $[17,28,29,30,31,32,33]$. Although only the mature osteoblasts participate actively in bone production, many different differentiation stages of osteoblasts are present in bone tissue.

In the present investigation we hypothesised that there is mutual interplay between prostate carcinoma cells and the predominant bone cell type: the osteoblast. In order to examine the effect of 
osteoblasts on prostate cancer cells we established an artificial coculture model allowing the exchange of soluble chemical substances between the two cell types. After the co-culture period, we examined possible gene expression changes induced by the medium contact. A large number of changes in the prostate cancer cells thus may indicate a complex collaboration between cancer cells and host microenvironment in bone.

A main point in our investigation is the observation that differentiation status in osteoblasts plays a crucial role for the PC-3 and DU-145 gene expression pattern produced by co-culturing, even though the differentiation status in osteoblasts influence the two cell lines in a different manner. However, no expressional changes associated with apoptosis in DU-145 could be found, which indicate that the PC-3 cells (originally isolated from a bone metastasis) seems to be more sensitive to ant-apoptotic signaling produced by differentiated ostoblast than the brain lesion cell line DU-145. We also demonstrate that the choice of array platform protocol is crucial for the total number of gene transcript differential expressed. However, it is of very importance that the GO- term "caspase regulation" is common for both platforms, which indicates that the picture drawn is biologically representative, rather than a technical artefact.

Very few studies that have investigated this type of interactions have described differentiation of osteoblasts, and specifically established the mature bone-producing phenotype. Our results points out the matter of necessity to strictly determine the osteoblasts differentiation status before co-culturing such cells with prostate epithelia. It seems clear that it is very important to do this determination, especially when using hFOB1.19 which have been shown to possess stem cell-like characteristics and may reflect a more osteoprogenitor-like phenotype. Based on the differences in experimental designs between our study and similar investigations, it is difficult to compare our results with other studies due to the use of differentiated phenotype. However, our results point out that several changes influence the ability of prostate cancer cells to survive, in respect to the observed repression of positive regulators of apoptosis. Our results also show that genes relevant to cell-matrix adhesion processes are considerable affected by the co-culturing with differentiated osteoblasts. Interestingly, previous studies suggest that the spread of prostate cancer to bone is supported by altered expression of specific cell adhesion molecules [34,35].

Because of the complexity of the metastasis process, it is a huge challenge to elucidate the interplay between prostate carcinoma cells and their favourite homing site in the skeletal system. Currently, experiments are in progress to determine how cells translate the changes in gene expression patterns into phenotypic alterations. Hopefully, the increased knowledge about malignant epithelial cells' behaviour will open doors for new treatment strategies against the cancer's primary characteristic, namely its ability to invade into neighbouring tissue and secondarily its ability to form distant metastasis. Obviously, further studies are needed to throw light on these processes, but the result reported in this publication gives strength to the hypothesis that interactions between prostate cancer cells and mature bone-producing osteoblasts might be potential targets when developing new cancer therapy strategies.

\section{References}

1. Morrissey C, Vessella RL (2007) The role of tumor microenvironment in prostate cancer bone metastasis. J Cell Biochem 101: 873-886.

2. Pienta KJ, Loberg R (2005) The "emigration, migration, and immigration" of prostate cancer. Clin Prostate Cancer 4: 24-30.
3. Vessella RL, Corey E (2006) Targeting factors involved in bone remodeling as treatment strategies in prostate cancer bone metastasis. Clin Cancer Res 12: 6285s-6290s.

4. Clark PE, Torti FM (2003) Prostate cancer and bone metastases: medical treatment. Clin Orthop Relat Res S1 48- S157.

5. Tantivejkul K, Kalikin LM, Pienta KJ (2004) Dynamic process of prostate cancer metastasis to bone. J Cell Biochem 91: 706-717.

6. Keller ET (2002a) Overview of metastasis and metastases. J Musculoskel Neuron Interact 2: 567-569.

7. Liao J, Schneider A, Datta NS, McCauley LK (2006) Extracellular calcium as a candidate mediator of prostate cancer skeletal metastasis. Cancer Res 66 : 9065-9073.

8. Jacob K, Webber M, Benayahu D, Kleinman HK (1999) Osteonectin promotes prostate cancer cell migration and invasion: a possible mechanism for metastasis to bone. Cancer Res 59: 4453-4457.

9. Festuccia C, Bologna M, Gravina GL, Guerra F, Angelucci A, et al. (1999) Osteoblast conditioned media contain TGF-beta1 and modulate the migration of prostate tumor cells and their interactions with extracellular matrix components. Int J Cancer 81: 395-403.

10. Rajan R, Vanderslice R, Kapur S, Lynch J, Thompson R, et al. (1996) Epiderma growth factor (EGF) promotes chemomigration of a human prostate tumor cell line, and EGF immunoreactive proteins are present at sites of metastasis in the stroma of lymph nodes and medullary bone. Prostate 28: 1-9.

11. Ritchie CK, Andrews LR, Thomas KG, Tindall DJ, Fitzpatrick LA (1997) The effects of growth factors associated with osteoblasts on prostate carcinoma proliferation and chemotaxis: implications for the development of metastatic disease. Endocrinology 138: 1145-1150.

12. Zlotnik A (2004) Chemokines in neoplastic progression. Semin Cancer Biol 14 181-185.

13. Zlotnik A (2006) Chemokines and cancer. International Journal of Cancer 119 2026-2029.

14. Taichman RS, Cooper C, Keller ET, Pienta KJ, Taichman NS, et al. (2002) Use of the stromal cell-derived factor-1/CXCR4 pathway in prostate cancer metastasis to bone. Cancer Res 62: 1832-1837.

15. Scott LJ, Clarke NW, George NJ, Shanks JH, Testa NG, et al. (2001) Interactions of human prostatic epithelial cells with bone marrow endothelium: binding and invasion. Br J Cancer 84: 1417-1423.

16. Koeneman KS, Yeung F, Chung LW (1999) Osteomimetic properties of prostate cancer cells: a hypothesis supporting the predilection of prostate cancer metastasis and growth in the bone environment. Prostate 39: 246-261.

17. Knerr K, Ackermann K, Neidhart T, Pyerin W (2004) Bone metastasis: Osteoblasts affect growth and adhesion regulons in prostate tumor cells and provoke osteomimicry. Int J Cancer 111: 152-159.

18. Kaighn ME, Narayan KS, Ohnuki Y, Lechner JF, Jones LW (1979) Establishment and characterization of a human prostatic carcinoma cell line (PC-3). Invest Urol 17: 16-23.

19. Harris SA, Enger RJ, Riggs BL, Spelsberg TC (1995) Development and characterization of a conditionally immortalized human fetal osteoblastic cell line. J Bone Miner Res 10: 178-186.

20. Chung CH, Golub EE, Forbes E, Tokuoka T, Shapiro IM (1992) Mechanism of action of beta-glycerophosphate on bone cell mineralization. Calcif Tissue Int 51: 305-311.

21. Franceschi RT, Iyer BS, Cui Y (1994) Effects of ascorbic acid on collagen matrix formation and osteoblast differentiation in murine MC3T3-E1 cells. J Bone Miner Res 9: 843-854.

22. Hayami T, Zhang Q, Kapila Y, Kapila S (2007) Dexamethasone's enhancement of osteoblastic markers in human periodontal ligament cells is associated with inhibition of collagenase expression. Bone 40: 93-104.

23. Standal T, Abildgaard N, Fagerli UM, Stordal B, Hjertner O, et al. (2007) HGF inhibits BMP-induced osteoblastogenesis: possible implications for the bone disease of multiple myeloma. Blood 109: 3024-3030.

24. Irizarry RA, Bolstad BM, Collin F, Cope LM, Hobbs B, et al. (2003) Summaries of Affymetrix Gene Chip probe level data. Nucleic Acids Res. 31: e15.

25. Gidskehaug L, Anderssen E, Flatberg A, Alsberg BK (2007) A framework for significance analysis of gene expression data using dimension reduction methods. BMC Bioinformatics 8: 346 . 
Citation: Skogseth H, Dybwad M, Flatberg A, Halgunset J (2010) Mature Bone-Producing Osteoblasts Alter Gene Expression of Metastasis Related Genes in Prostate Cancer Cells. J Carcinogene Mutagene 1:111. doi:10.4172/2157-2518.1000111

Page 7 of 7

26. Wettenhall JM, Smyth GK (2005) limmaGUI: a graphical user interface for linear modeling of microarray data. Bioinformatics 20: 3705-3706.

27. Beisvag V, Junge FK, Bergum H, Jolsum L, Lydersen S, et al. (2006) GeneTools-application for functional annotation and statistical hypothesis testing. BMC Bioinformatics 7: 470 .

28. Logothetis CJ, Lin SH (2005) Osteoblasts in prostate cancer metastasis to bone. Nat Rev Cancer 5: 21-28.

29. Pinski J, Parikh A, Bova GS, Isaacs JT (2001) Therapeutic implications of enhanced $\mathrm{G}(0) / \mathrm{G}(1)$ checkpoint control induced by coculture of prostate cancer cells with osteoblasts. Cancer Res 61: 6372-6376.

30. Fizazi K, Yang J, Peleg S, Sikes CR, Kreimann EL, et al. (2003) Prostate cancer cells-osteoblast interaction shifts expression of growth/survival-related genes in prostate cancer and reduces expression of osteoprotegerin in osteoblasts. Clin Cancer Res 9: 2587-2597.

31. Yang J, Fizazi K, Peleg S, Sikes CR, Raymond AK, et al. (2001) Prostate cancer cells induce osteoblast differentiation through a Cbfa1-dependent pathway. Cancer Res 61: 5652-5659.
32. Fu Z, Dozmorov IM, Keller ET (2002) Osteoblasts produce soluble factors that induce a gene expression pattern in non-metastatic prostate cancer cells, similar to that found in bone metastatic prostate cancer cells. Prostate 51: 1020.

33. Axanova, L, Morré DJ, Morré DM (2005) Growth of LNCaP cells in monoculture and coculture with osteoblasts and response to tNOX inhibitors. Cancer Lett 225: 35-40.

34. Bryden AA, Hoyland JA, Freemont AJ, Clarke NW, Schembri Wismayer D, et al. (2002) E-cadherin and $\beta$-catenin are down-regulated in prostatic bone metastases. BJU Int 89: 400-403.

35. Fidler IJ, Kim SJ, Langley RR (2007) The role of the organ microenvironment in the biology and therapy of cancer metastasis. J Cell Biochem 101: 927-936.

36. Keller ET (2002) The role of osteoclastic activity in prostate cancer skeletal metastases. Drugs Today (Barc) 38: 91-102.

37. Lu Y, Cai Z, Galson DL, Xiao G, Liu Y, et al. (2006) Monocyte chemotactic protein-1 (MCP-1) acts as a paracrine and autocrine factor for prostate cancer growth and invasion. Prostate 66: 1311-1318. 